\title{
Fabrication and characterization of carbon-based flexible strain sensor
}

\begin{abstract}
This research covers carbon-based strain sensors which are designed and printed onto flexible substrates. Conductive nanocomposite of Polydimethylsiloxane (PDMS) and Carbon Black (CB) powder with $10 \%$ of $\mathrm{CB}$ have been used as the sensing element utilizing its flexibility and conductivity while silver conductive paste is used as the electrode for the strain sensor. Several designs with different length of strain sensors were printed using screen printing technology onto $0.75 \varepsilon \mathrm{m}$-Kapton $\mathrm{HN}$ film. Printed sensors were then cured at $85^{\circ} \mathrm{C}$ (for carbon printing) and $145^{\circ} \mathrm{C}$ (for silver printing). Parameters characterized are resistivity, conductivity and stretchability of the sensor using tensile test. The relationship between force, strain and resistance for all sensors designed are also discussed. The measured resistivity and conductivity of the sensor are $0.43 \mathrm{qm}$ and $2.4 \mathrm{~S} / \mathrm{m}$ respectively while the stretchability of the sensor on Kapton film has a maximum strain of $45 \%$. This flexible strain sensor is very useful in many applications such as health monitoring systems, human motion detection and many more.
\end{abstract}

Keyword: Flexible electronics; Sensor; Strain sensor; Carbon-based; Conductive paste 